\title{
Multivariate Fuss-Narayana polynomials and their application to random matrices
}

\author{
Romuald Lenczewski \\ Instytut Matematyki i Informatyki \\ Politechnika Wrocławska \\ Wrocław, Poland \\ romuald.lenczewski@pwr.wroc.pl
}

\author{
Rafał Sałapata \\ Instytut Matematyki i Informatyki \\ Politechnika Wrocławska \\ Wrocław, Poland \\ rafal.salapata@pwr.wroc.pl
}

Submitted: Oct 12, 2012; Accepted: May 24, 2013; Published: May 31, 2013

Mathematics Subject Classifications: 05A15, 15B52, 46L54

\begin{abstract}
It has been shown recently that the limit moments of $W(n)=B(n) B^{*}(n)$, where $B(n)$ is a product of $p$ independent rectangular random matrices, are certain homogeneous polynomials $P_{k}\left(d_{0}, d_{1}, \ldots, d_{p}\right)$ in the asymptotic dimensions of these matrices. Using the combinatorics of noncrossing partitions, we explicitly determine these polynomials and show that they are closely related to polynomials which can be viewed as multivariate Fuss-Narayana polynomials. Using this result, we compute the moments of $\varrho_{t_{1}} \otimes \varrho_{t_{2}} \otimes \ldots \otimes \varrho_{t_{m}}$ for any positive $t_{1}, t_{2}, \ldots, t_{m}$, where $\nabla$ is the free multiplicative convolution in free probability and $\varrho_{t}$ is the Marchenko-Pastur distribution with shape parameter $t$.
\end{abstract}

Keywords: Fuss-Narayana numbers, Narayana polynomials, Marchenko-Pastur law, free probability, random matrix

\section{Introduction}

The motivation of this paper comes from some recent developments concerning random matrices and their products [8], where combinatorial formulas for the polynomials studied in this paper were obtained.

For any given $p \in \mathbb{N}$ and any $n \in \mathbb{N}$, consider the product of independent rectangular Gaussian random matrices

$$
B(n)=X_{1}(n) X_{2}(n) \ldots X_{p}(n),
$$

where $n \in \mathbb{N}$ and their dimensions are such that the product is well-defined. If $X_{j}$ is an $N_{j-1}(n) \times N_{j}(n)$ matrix for any $1 \leqslant j \leqslant p$, we assume that

$$
\lim _{n \rightarrow \infty} N_{j}(n) / n=d_{j}>0,
$$


where $d_{0}, d_{1}, \ldots d_{p}$ are called asymptotic dimensions. This notation slightly differs from $d_{1}, d_{2}, \ldots, d_{p+1}$ used in [8], but it is more convenient in combinatorial formulas. Finally, let $\tau_{0}(n)$ be the trace over the set of $N_{0}(n)$ basis vectors composed with classical expectation.

It is shown in [8] that under certain natural assumptions

$$
\lim _{n \rightarrow \infty} \tau_{0}(n)\left(\left(B(n) B^{*}(n)\right)^{k}\right)=P_{k}\left(d_{0}, d_{1}, \ldots, d_{p}\right),
$$

where on the right-hand side we have a certain homogeneous polynomial of order $p k$ for any $k \in \mathbb{N}$. There, instead of the matrices $X_{1}(n), X_{2}(n), \ldots, X_{p}(n)$, symmetric blocks embedded in a large $n \times n$ square matrix were used and $\tau_{0}(n)$ was the partial trace over a subset of basis vectors composed with classcial expectation. That formulation followed from the fact that it was the ensemble of symmetric blocks which was shown to converge in moments to the ensemble of operators generalizing free Gaussian operators which were symmetrizations of matricially free Gaussian operators.

Non-homogeneous polynomials obtained from $P_{k}$ by dividing them by $d_{0}^{k p}$ were called 'generalizations of Narayana polynomials' since for $p=1$ they become the well-known Narayana polynomials of one variable corresponding to Catalan numbers and their decompositions in terms of Narayana numbers. In random matrix theory, these well-known combinatorial objects are important since they are related to the moments of the MarchenkoPastur distribution, the limit distribution of Wishart random matrices [10].

In the case when $p>1$ and all matrices in the product are square, the limit moments are Fuss-Narayana polynomials of one variable. These polynomials correspond, in turn, to Fuss-Catalan numbers and their decompositions in terms of Fuss-Narayana numbers. For arbitrary asymptotic dimensions, it is therefore natural to expect that we should obtain some multivariate analogs of Fuss-Narayana polynomials.

A combinatorial definition of the polynomials $P_{k}$ was given in [8]. In this paper, we determine their explicit form, using purely combinatorial methods. Namely, we show that

$$
P_{k}\left(d_{0}, d_{1}, \ldots, d_{p}\right)=\sum_{j_{0}+\ldots+j_{p}=p k+1} \frac{1}{k}\left(\begin{array}{l}
k \\
j_{0}
\end{array}\right)\left(\begin{array}{l}
k \\
j_{1}
\end{array}\right) \ldots\left(\begin{array}{l}
k \\
j_{p}
\end{array}\right) d_{0}^{j_{0}-1} d_{1}^{j_{1}} \ldots d_{p}^{j_{p}},
$$

where the indices $j_{0}, j_{1}, \ldots, j_{p}$ are natural numbers. When we divide these polynomials by $d_{0}^{k p}$, which corresponds to a different normalization of random matrices, we obtain

$$
F_{k}\left(t_{1}, t_{2}, \ldots, t_{p}\right)=d_{0}^{-k p} P_{k}\left(d_{0}, d_{1}, \ldots, d_{p}\right),
$$

where $t_{j}=d_{j} / d_{0}$ for any $j$, called multivariate Fuss-Narayana polynomials since for $p=1$ they become the well-known Narayana polynomials.

The coefficients built from binomial expressions play the role of generalized FussNarayana numbers. Such numbers appeared recently in a different context in the paper of Loktev on Weyl modules of Lie algebras [9], who showed that they were dimensions of weight spaces of two-variable Weyl modules of Lie algebras $g l_{p+1}$.

In random matrix theory, Fuss-Catalan numbers appeared in the paper of Alexeev, Goetze and Tikhomirov [1], who showed, using the methods of classical probability, that 
they were the limit moments of the asymptotic distribution of squared singular values of powers of a random matrix with independent entries. Moreover, similar techniques were used by the same authors to study the case of products of independent random matrices [2]. In the general case of rectangular matrices, the limit distribution for singular values of such products has only been described by an algebraic equation for its CauchyStieltjes transform and a formula for moments has not been derived. Our approach is quite different since we use noncommutative probability and operator algebras. This allows us to formulate the problem in a purely combinatorial fashion and then solve it using combinatorial techniques.

An important probabilistic context in which Fuss-Narayana polynomials of one variable appear is that of free Bessel laws

$$
\pi_{p, t}=\varrho_{1}^{\bigotimes(p-1)} \otimes \varrho_{t},
$$

where $p \in \mathbb{N}, \varrho_{t}$ is the Marchenko-Pastur distribution with shape parameter $t>0$, and $\otimes$ denotes the free multiplicative convolution. They were defined by Banica, Belinschi, Capitaine and Collins [3], who have shown that their moments are given by Fuss-Narayana polynomials in $t$.

A more general multiplicative free convolution of Marchenko-Pastur laws is of the form

$$
\varrho_{t_{1}} \otimes \varrho_{t_{2}} \otimes \ldots \otimes \varrho_{t_{p}}
$$

for any positive $t_{1}, t_{2}, \ldots, t_{p}$. We show in this paper that the moments of such convolutions are given by multivariate Fuss-Narayana polynomials. For the foundations of free probability and, in particular, for the definition of the free multiplicative convolution, see $[11]$.

The paper is organized as follows. In Section 2, we introduce multivariate Narayana polynomials $F_{k}$ and their coefficients called generalized Fuss-Narayana numbers. In Section 3, we prove two lemmas on a family of generating functions, among which is the generating function defined by the polynomials $P_{k}$ which are limit moments of certain random matrices. These lemmas enable us to prove in Section 4 that the polynomials $P_{k}$ are closely related to multivariate Fuss-Narayana polynomials. Finally, we show that the latter give the moments of multiplicative free convolutions of Marchenko-Pastur laws.

\section{Multivariate Fuss-Narayana polynomials}

The Fuss-Catalan numbers associated with $p \in \mathbb{N}$ are given by the formula

$$
C_{k}=\frac{1}{k}\left(\begin{array}{c}
(p+1) k \\
p k+1
\end{array}\right),
$$

where $k \in \mathbb{N}$. The following decomposition of Fuss-Catalan numbers is known as the generalized Vandermonde's identity. 
Proposition 1. For any $p, k \in \mathbb{N}$, the following decomposition hold:

$$
C_{k}=\sum_{j_{0}+\ldots+j_{p}=p k+1} N\left(k, j_{0}, \ldots, j_{p}\right)
$$

where the summation runs over all $j_{0}, \ldots, j_{p} \in[k]=\{1,2, \ldots, k\}$ for which it holds that $j_{0}+\ldots+j_{p}=p k+1$, and for such values

$$
N\left(k, j_{0}, \ldots, j_{p}\right)=\frac{1}{k}\left(\begin{array}{c}
k \\
j_{0}
\end{array}\right)\left(\begin{array}{c}
k \\
j_{1}
\end{array}\right) \ldots\left(\begin{array}{c}
k \\
j_{p}
\end{array}\right) .
$$

These numbers will be called generalized Fuss-Narayana numbers.

Proof. If we apply the Vandermonde's identity to the formula for the Fuss-Catalan numbers $p$ times, we obtain the above formula.

Note that after the first application of the Vandermonde's identity, we get the decomposition

$$
C_{k}=\sum_{j_{0}+j_{1}=p k+1} \frac{1}{k}\left(\begin{array}{c}
k \\
j_{0}
\end{array}\right)\left(\begin{array}{c}
p k \\
j_{1}
\end{array}\right),
$$

where the summands on the right-hand side are called Fuss-Narayana numbers.

It will be convenient to use vector notations

$$
\mathbf{d}=\left(d_{0}, d_{1}, \ldots, d_{p}\right), \mathbf{j}=\left(j_{0}, j_{1}, \ldots, j_{p}\right),
$$

where $d_{0}, d_{1}, \ldots, d_{p}$ are variables and $j_{0}, j_{1}, \ldots, j_{p}$ are nonnegative integers. Using these vectors to write the generalized Fuss-Narayana numbers as $N(k, \mathbf{j})$ and generalized powers in the form

$$
\mathbf{d}^{\mathbf{j}}=d_{0}^{j_{0}} d_{1}^{j_{1}} \ldots d_{p}^{j_{p}}
$$

we can define the generating function for the generalized Fuss-Narayana numbers by

$$
\mathcal{N}(x, \mathbf{d})=\sum_{k=1}^{\infty} \sum_{j_{0}+\ldots+j_{p}=p k+1} N(k, \mathbf{j}) \mathbf{d}^{\mathbf{j}} x^{k} .
$$

We will set $N(k, \mathbf{j})=0$ whenever $j_{0}+\ldots+j_{p} \neq p k+1$ or $j_{i} \notin[k]$ for some $i$.

Dividing the homogeneous polynomials in $p+1$ asymptotic dimensions of the form

$$
R_{k}\left(d_{0}, d_{1}, \ldots, d_{p}\right)=\sum_{j_{0}+\ldots+j_{p}=p k+1} N(k, \mathbf{j}) d_{0}^{j_{0}} d_{1}^{j_{1}} \ldots d_{p}^{j_{p}}
$$

by $d_{0}^{p k+1}$, we obtain certain non-homogeneous polynomials in $p$ variables $t_{i}=d_{i} / d_{0}$, where $i \in[p]$, which play the role of mutlivariate generalizations of Narayana polynomials, obtained for $p=1$. 
Definition 2. Non-homogeneous polynomials of $p$ variables of the form

$$
F_{k}\left(t_{1}, t_{2}, \ldots, t_{p}\right)=\sum_{j_{0}+\ldots+j_{p}=p k+1} N\left(k, j_{0}, \ldots, j_{p}\right) t_{1}^{j_{1}} t_{2}^{j_{2}} \ldots t_{p}^{j_{p}},
$$

where $k \in \mathbb{N}$, will be called multivariate Fuss-Narayana polynomials.

Remark 3. Of course, one can also obtain these polynomials from $R_{k}$ by setting $d_{0}=1$ and $d_{j}=t_{p}$ for $j \in[p]$. In fact, there is a number of equivalent definitions of this type, namely

$$
F_{k}\left(t_{1}, t_{2}, \ldots, t_{p}\right)=R_{k}\left(1, t_{1}, \ldots, t_{p}\right)=\ldots=R_{k}\left(t_{1}, t_{2}, \ldots, t_{p}, 1\right),
$$

due to the symmetric form of generalized Fuss-Narayana numbers.

In order to establish a formula satisfied by the generating function $\mathcal{N}$, we will use the next theorem, which is a special version of the well-known Lagrange Inversion Theorem (see, for instance, Appendix A in the paper of Deutch [4] or the book of Wilf [12]).

Theorem 4. Assume that a generating function $f(z)$ satisfies the functional equation

$$
f(z)=z H(f(z)),
$$

where $H$ is a polynomial. Then (1) has a unique solution and

$$
\left[z^{n}\right] f(z)=\frac{1}{n}\left[\lambda^{n-1}\right] H^{n}(\lambda),
$$

where $\left[z^{n}\right] f(z)$ is the coefficient of the series $f(z)$ standing by $z^{n}$.

Proposition 5. For any $d_{0}, \ldots, d_{p}$, the unique solution of the equation

$$
g(x)=x \prod_{i=0}^{p}\left(g(x)+d_{i}\right)
$$

is given by $g(x)=\mathcal{N}(x, \mathbf{d})$.

Proof. Applying Theorem 4 to the polynomial $H(\lambda)=\left(\lambda+d_{0}\right)\left(\lambda+d_{1}\right) \ldots\left(\lambda+d_{p}\right)$, we get existence of a unique solution $g(x)$ of equation (2). We must have

$$
\begin{aligned}
{\left[x^{n}\right] g(x) } & =\frac{1}{n}\left[\lambda^{n-1}\right]\left(\prod_{i=0}^{p}\left(\lambda+d_{i}\right)\right)^{n} \\
& =\frac{1}{n}\left[\lambda^{n-1}\right] \prod_{i=0}^{p}\left(\sum_{k=0}^{n}\left(\begin{array}{l}
n \\
k
\end{array}\right) \lambda^{k} d_{i}^{n-k}\right) \\
& =\frac{1}{n}\left[\lambda^{n-1}\right] \sum_{k=0}^{\infty}\left(\sum_{k_{0}+\ldots+k_{p}=k}\left(\begin{array}{c}
n \\
k_{0}
\end{array}\right) \ldots\left(\begin{array}{c}
n \\
k_{p}
\end{array}\right) d_{0}^{n-k_{0}} \ldots d_{p}^{n-k_{p}}\right) \lambda^{k} \\
& =\frac{1}{n} \sum_{j_{0}+\ldots+j_{p}=p n+1}\left(\begin{array}{c}
n \\
j_{0}
\end{array}\right) \ldots\left(\begin{array}{c}
n \\
j_{p}
\end{array}\right) d_{0}^{j_{0}} \ldots d_{p}^{j_{p}},
\end{aligned}
$$

where we put $n-k_{i}=j_{i}$ for $i \in\{0,1, \ldots, p\}$ to get the last equation. This proves that $g(x)=\mathcal{N}(x, \mathbf{d})$. 


\section{Lemmas}

In this section, we shall prove two combinatorial lemmas, in which we compare the generating function $\mathcal{N}$ of Section 2 defined by polynomials $R_{k}$ with the generating function $\mathcal{N}_{0}$ defined by polynomials $P_{k}$. Let us remark that we shall use similar letters in our notations: $\mathcal{N}$ and $\mathcal{N}_{j}$ will denote generating functions, whereas $N$ and $N_{j}$, respectively, will be their coefficients (usually, both symbols will be followed by some arguments).

By a noncrossing pair partition of the set $[m]:=\{1,2, \ldots, m\}$, where $m=2 k$ is an even natural number, we understand a collection $\pi=\left\{\pi_{1}, \pi_{2}, \ldots, \pi_{k}\right\}$ of disjoint twoelement subsets of $[m]$ called blocks, such that there are no blocks $\{i, j\}$ and $\{p, q\}$ for which $i<p<j<q$. The set of such partitions will be denoted by $\mathcal{N C}_{m}^{2}$. If $\{i, j\}$ is a block and $i<j$, then $i$ and $j$ are called the left and the right legs of this block, respectively. For a given noncrossing pair partition $\pi$, we denote by $\mathcal{R}(\pi)$ the set of its right legs.

We will consider certain noncrossing pair-partitions of $[m]$, where $m=2 p k$ and $p, k \in$ $\mathbb{N}$, which are associated with words of the form

$$
W_{0}^{k}=\left(1 \ldots p p^{*} \ldots 1^{*}\right)^{k}
$$

built from $p$ starred and $p$ unstarred letters. More generally, we will denote by $W_{i}$ the word that arises from $W_{0}$ by the cyclic shift of its letters to the right by $i$ positions, where $i \in\{0,1, \ldots, p\}$, namely

$$
W_{i}=i^{*} \ldots 1^{*} 1 \ldots p p^{*} \ldots(i+1)^{*}
$$

thus, in particular, $W_{p}=p^{*} \ldots 1^{*} 1 \ldots p$. We will also consider powers $W_{i}^{k}$ of such shifted words. For simplicity, we supress $p$ in all these notations, in contrast to the notation used in $[8]$.

Definition 6. We shall say that $\pi \in \mathcal{N C}_{2 p k}^{2}$ is adapted to the word $W_{i}^{k}$ if all its blocks are associated with pairs of letters of the form $\left\{l, l^{*}\right\}$ for some $l \in\{1, \ldots, p\}$. By $\mathcal{N C}_{2 p k}^{2}\left(W_{i}^{k}\right)$ we denote the set of all pair partitions from $\mathcal{N C}_{2 p k}^{2}$ which are adapted to $W_{i}^{k}$. We set $\mathcal{N C}_{0}^{2}\left(W_{i}^{0}\right)=\{\emptyset\}$

Thus, if $\pi \in \mathcal{N C}_{2 p k}^{2}\left(W_{i}^{k}\right)$, then blocks of $\pi$ are pairs $\{r, s\}$ in which $r$ is associated with the letter $l$ if and only if $s$ is associated with the letter $l^{*}$. Thus, if $r<s$, then $s$ is the right leg of this block and it is associated with $l^{*}$, whereas if $r>s$, then $r$ is the right leg of this block and it is associated with $l$. Therefore, it is meaningful to define the sets of right legs of $\pi$ associated with $l$ and $l^{*}$, respectively, and denote these sets by $\mathcal{R}_{l}(\pi)$ and $\mathcal{R}_{l}^{*}(\pi)$. Using these sets, whose union gives $\mathcal{R}(\pi)$, we defined in [8] the following family of homogeneous polynomials in $p+1$ variables.

Definition 7. Define polynomials in variables $d_{0}, d_{1}, \ldots, d_{p}$ of the form

$$
P_{k}\left(d_{0}, d_{1}, \ldots, d_{p}\right)=\sum_{\pi \in \mathcal{N} \mathcal{C}_{2 k p}^{2}\left(W_{0}^{k}\right)} d_{0}^{j_{0}(\pi)} d_{1}^{j_{1}(\pi)} \ldots d_{p}^{j_{p}(\pi)}
$$


for any $k, p \in \mathbb{N}$, where

$$
j_{l}(\pi)=\left|\mathcal{R}_{l+1}(\pi)\right|+\left|\mathcal{R}_{l}^{*}(\pi)\right|
$$

for any $\pi \in \mathcal{N C}_{2 k p}^{2}\left(W_{0}^{k}\right)$ and $0 \leqslant l \leqslant p$, where we set $\mathcal{R}_{0}^{*}(\pi)=\emptyset$ and $\mathcal{R}_{p+1}(\pi)=\emptyset$.

Our goal is now to count the number of noncrossing pair partitions $\pi \in \mathcal{N C}_{2 k p}^{2}\left(W_{0}^{k}\right)$ which contribute identical monomials to the polynomials $P_{k}$. In other words, if we label the legs of $\pi$ by letters from the set $\left\{1, \ldots, p, p^{*}, \ldots, 1^{*}\right\}$, we would like to count the blocks labelled by ordered pairs $\left(l, l^{*}\right)$ or $\left((l+1)^{*},(l+1)\right)$ for any $l \in\{0,1, \ldots, p\}$.

We are mainly interested in counting the noncrossing pair partitions adapted to $W_{0}^{k}$ which contribute identical monomials, but we shall need a family which encodes detailed information about the labellings of their right legs, namely

$$
N_{i}(k, \mathbf{j})=\#\left\{\pi \in \mathcal{N C}_{2 p k}^{2}\left(W_{i}^{k}\right) ; j_{l}(\pi)=j_{l} \text { for } l \in\{0,1, \ldots, p\}\right\},
$$

where $\mathbf{j}=\left(j_{0}, j_{1}, \ldots, j_{p}\right)$ and $i \in\{0,1, \ldots, p\}$. It is obvious that

$$
N_{i}(k, \mathbf{j})=0 \text { whenever } j_{0}+\ldots+j_{p} \neq p k \text { or } j_{i}>k \text { for some } i,
$$

and that $N_{i}(0,0, \ldots, 0)=1$ for any $i$. Let us add that the reason why we count together right legs labelled by $l^{*}$ and $l+1$ follows from the way we multiply rectangular matrices and their adjoints and is clear from the proof of [8, Theorem 10.1].

The corresponding generating functions are given by

$$
\mathcal{N}_{i}(x, \mathbf{d})=\sum_{k, j_{0}, \ldots, j_{p}=0}^{\infty} N_{i}(k, \mathbf{j}) \mathbf{d}^{\mathbf{j}} x^{k} .
$$

We are especially interested in the coefficients of the generating function for $i=0$, i.e. we will study homogeneous polynomials of degrees $p k$ in variables $d_{0}, d_{1}, \ldots, d_{p}$ given by the combinatorial formulas

$$
P_{k}\left(d_{0}, d_{1}, \ldots, d_{p}\right)=\sum_{\pi \in \mathcal{N} C_{2 p k}^{2}\left(W_{0}^{k}\right)} d_{0}^{j_{0}(\pi)} d_{1}^{j_{1}(\pi)} \ldots d_{p}^{j_{p}(\pi)}=\sum_{j_{0}, \ldots, j_{p}=0}^{\infty} N_{0}(k, \mathbf{j}) \mathbf{d}^{\mathbf{j}}
$$

for $k \geqslant 0$, where, in the last formula, only finitely many terms do not vanish. Obviously,

$$
\mathcal{N}_{0}(x, \mathbf{d})=\sum_{k=0}^{\infty} P_{k}\left(d_{0}, d_{1}, \ldots, d_{p}\right) x^{k} .
$$

Our goal in this section is to find the coefficients $N_{0}(k, \mathbf{j})$ of the polynomials $P_{k}$ and show that they are generalized Fuss-Narayana numbers. Moreover, if we divide $P_{k}$ by $d_{0}^{k p}$, we will obtain multivariate Fuss-Narayana polynomials of $t_{1}=d_{1} / d_{0}, \ldots, t_{p}=d_{p} / d_{0}$.

Example 8. Consider the case $p=2$. Then, there are three noncrossing pair partitions which are adapted to the word $W_{0}^{2}=\left(122^{*} 1^{*}\right)^{2}$, corresponding to the diagrams 

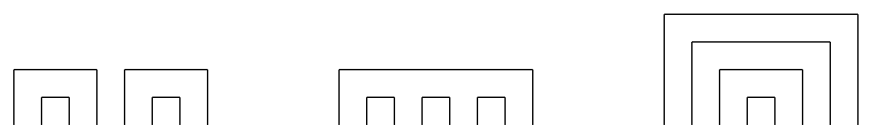

and thus

$$
P_{2}\left(d_{0}, d_{1}, d_{2}\right)=d_{1}^{2} d_{2}^{2}+d_{0} d_{1} d_{2}^{2}+d_{0} d_{1}^{2} d_{2}
$$

is the associated polynomial. If we take $W_{0}^{3}=\left(122^{*} 1^{*}\right)^{3}$, then the number of adapted noncrossing pair partitions grows to 12, which will coincide with the corresponding FussCatalan number of Corollary 14. Let us just give the corresponding polynomial

$$
P_{3}\left(d_{0}, d_{1}, d_{2}\right)=d_{1}^{3} d_{2}^{3}+3 d_{0} d_{1}^{2} d_{2}^{3}+3 d_{0} d_{1}^{3} d_{2}^{2}+d_{0}^{2} d_{1} d_{2}^{3}+3 d_{0}^{2} d_{1}^{2} d_{2}^{2}+d_{0}^{2} d_{1}^{3} d_{2}
$$

since drawing all the diagrams seems too elaborate.

For further purposes, we introduce a function $\varphi: \mathcal{N C}_{2 p k}^{2} \rightarrow \mathcal{N C}_{2 p k}^{2}$ defined by the diagram

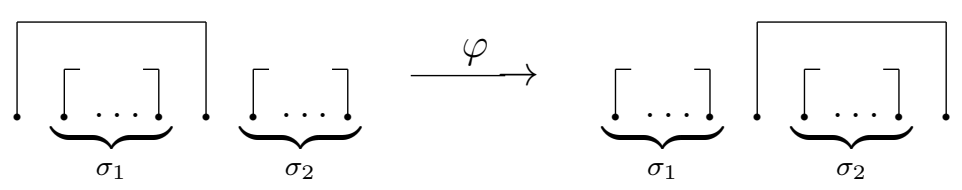

where $\sigma_{1} \in \mathcal{N C}_{2 k_{1}}^{2}, \sigma_{2} \in \mathcal{N C}_{2 k_{2}}^{2}$ and $k_{1}+k_{2}=p k-1$. The inverse of $\varphi$ is well-defined, which means that $\varphi$ is a bijection on $\mathcal{N C}_{2 p k}^{2}$. By $\varphi^{n}$ we denote the $n$-th composition of $\varphi$.

Lemma 9. For any $i \in\{1,2, \ldots, p\}$, it holds that

$$
d_{i}\left(\mathcal{N}_{i}(x, \mathbf{d})-1\right)=d_{0}\left(\mathcal{N}_{0}(x, \mathbf{d})-1\right) .
$$

Proof. First, we will show that

$$
N_{i}\left(k, j_{0}+1, j_{1}, \ldots, j_{p}\right)=N_{0}\left(k, j_{0}, \ldots, j_{i-1}, j_{i}+1, j_{i+1}, \ldots, j_{p}\right) .
$$

Let $\pi \in \mathcal{N C}_{2 p k}^{2}\left(W_{i}^{k}\right)$. Then the partition $\pi$ given by the diagram (the legs are labelled only by the set of letters $\left\{1, \ldots, p, p^{*}, \ldots, 1\right\}$ and not by all consecutive numbers from the set $[2 k p])$

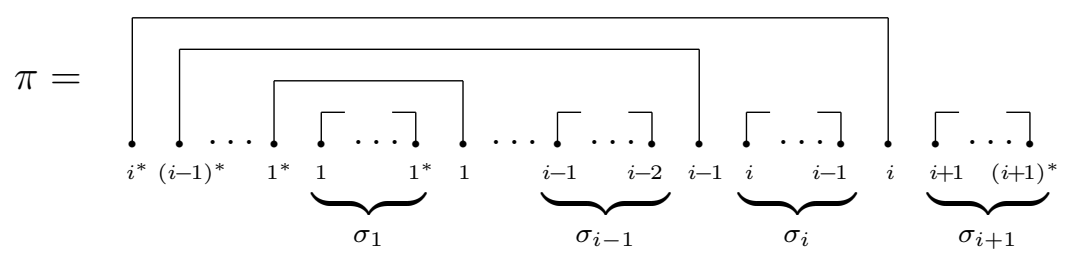

is mapped by $\varphi^{i}$ onto 


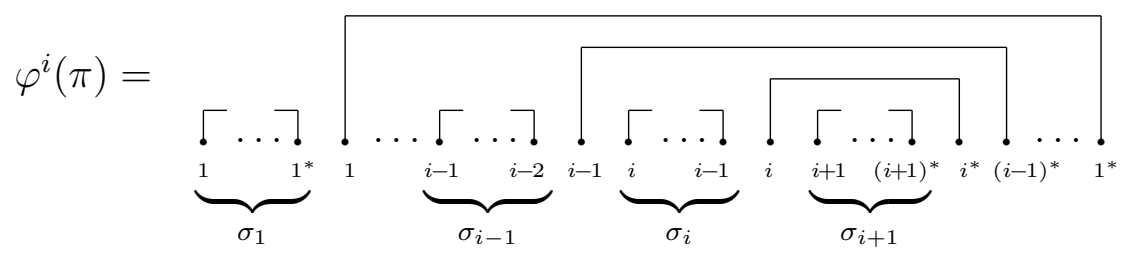

where $\sigma_{r} \in \mathcal{N C}_{2 k_{r}}^{2}$ and $k_{1}+\ldots+k_{i+1}=p k-i$. We understand that certain partitions among $\sigma_{1}, \ldots, \sigma_{i+1}$ in these diagrams may be empty. Obviously, $\varphi^{i}(\pi)$ is adapted to $W_{0}^{k}$, since $\pi$ is adapted to $W_{i}^{k}$ and, conversely, if $\pi$ wasn't adapted to $W_{i}^{k}$, then $\varphi^{i}(\pi)$ wouldn't be adapted to $W_{0}^{k}$. This means that $\varphi^{i}$ restricted to the set $\mathcal{N C}_{2 p k}^{2}\left(W_{i}^{k}\right)$ is a bijection between $\mathcal{N C}_{2 p k}^{2}\left(W_{i}^{k}\right)$ and $\mathcal{N C}_{2 p k}^{2}\left(W_{0}^{k}\right)$. Moreover, as compared with $\pi, \varphi^{i}(\pi)$ has one more block labelled by the ordered pair $\left(r, r^{*}\right)$ and one block fewer labelled by $\left(r^{*}, r\right)$ for each $r \in\{1, \ldots, i\}$. The numbers of other blocks are the same in $\pi$ and $\varphi^{i}(\pi)$. This means that

$$
j_{r}\left(\varphi^{i}(\pi)\right)=\left\{\begin{array}{ll}
j_{0}(\pi)-1 & \text { if } r=0 \\
j_{i}(\pi)+1 & \text { if } r=i \\
j_{i}(\pi) & \text { otherwise }
\end{array},\right.
$$

which proves (4). Now, using the fact that $j_{0}(\pi) \geqslant 1$ for $\pi \in \mathcal{N C}_{2 p k}^{2}\left(W_{i}^{k}\right)$ and $i, k \geqslant 1$, we get

$$
\begin{aligned}
d_{i}\left(\mathcal{N}_{i}(x, \mathbf{d})-1\right)= & d_{i} \sum_{k=1}^{\infty} \sum_{j_{0}=1}^{\infty} \sum_{j_{1}, \ldots, j_{p}=0}^{\infty} N_{i}\left(k, j_{0}, \ldots, j_{p}\right) d_{0}^{j_{0}} \ldots d_{p}^{j_{p}} x^{k} \\
= & d_{0} \sum_{k=1}^{\infty} \sum_{j_{0}, \ldots, j_{p}=1}^{\infty} N_{i}\left(k, j_{0}+1, \ldots, j_{p}\right) \\
& \times d_{0}^{j_{0}} \ldots d_{i-1}^{j_{i-1}} d_{i}^{j_{i}+1} d_{i+1}^{j_{i+1}} \ldots d_{p}^{j_{p}} x^{k} .
\end{aligned}
$$

Here, we use (4) and the observation that $j_{i}(\pi) \geqslant 1$ for $\pi \in \mathcal{N C}_{2 p k}^{2}\left(W_{0}^{k}\right)$ and $i, k \geqslant 1$. This becomes

$$
\begin{aligned}
d_{i}\left(\mathcal{N}_{i}(x, \mathbf{d})-1\right)= & d_{0} \sum_{k=1}^{\infty} \sum_{j_{0}, \ldots, j_{p}=0}^{\infty} N_{0}\left(k, j_{0}, \ldots, j_{i}, j_{i}+1, \ldots, j_{p}\right) \\
& \times d_{0}^{j_{0}} \ldots d_{i}^{j_{i}+1} \ldots d_{p}^{j_{p}} x^{k} \\
= & d_{0} \sum_{k=1}^{\infty} \sum_{j_{i}=1}^{\infty} \sum_{j_{0}, \ldots, j_{i-1}, j_{i+1} \ldots, j_{p}=0}^{\infty} N_{0}\left(k, j_{0}, \ldots, j_{i}, \ldots, j_{p}\right) \\
& \times d_{0}^{j_{0}} \ldots d_{i}^{j_{i}} \ldots d_{p}^{j_{p}} x^{k} \\
= & d_{0} \sum_{k=1}^{\infty} \sum_{j_{0}, \ldots, j_{p}=0}^{\infty} N_{0}(k, \mathbf{j}) \mathbf{d}^{\mathbf{j}} x^{k} \\
= & d_{0}\left(\mathcal{N}_{0}(x, \mathbf{d})-1\right),
\end{aligned}
$$

which completes the proof. 
Lemma 10. For any $p \in \mathbb{N}$, the generating functions $\mathcal{N}_{0}, \ldots, \mathcal{N}_{p}$ satisfy the equation

$$
\mathcal{N}_{0}(x, \mathbf{d})-1=x d_{1} \ldots d_{p} \prod_{i=0}^{p} \mathcal{N}_{i}(x, \mathbf{d}) .
$$

Proof. In order to prove this lemma, let us establish a recurrence relation between numbers $N_{i}(k, \mathbf{j})$ for $i \in\{0, \ldots, p\}$ and certain values of $k$ and $\mathbf{j}=\left(j_{0}, \ldots, j_{p}\right)$. For that purpose, observe that each partition $\pi \in \mathcal{N C}_{2 p k}^{2}\left(W_{0}^{k}\right)$ can be expressed in terms of the diagram

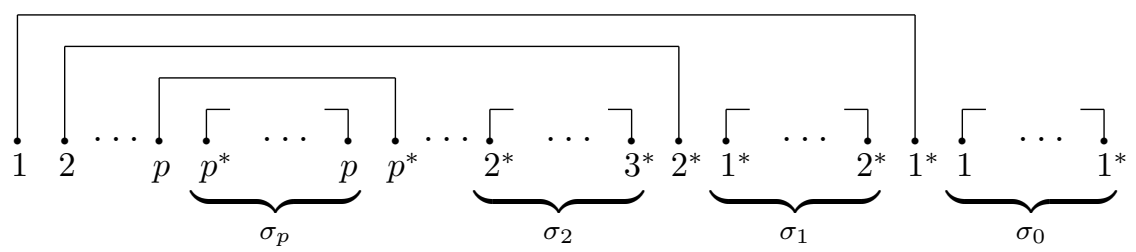

where $\sigma_{i} \in \mathcal{N C}_{2 p k_{i}}^{2}\left(W_{i}^{k_{i}}\right)$ and $k_{0}+k_{1}+\ldots+k_{p}=k-1$. We understand that each partition $\sigma_{i}$ in this diagram may be empty. Let $j_{i, r}=j_{r}\left(\sigma_{i}\right)$ be the number of blocks labelled by $\left(r, r^{*}\right)$ or $\left((r+1)^{*}, r+1\right)$ in $\sigma_{i}$. It will be convenient to denote by

$$
S_{r}(\pi)=j_{0, r}+j_{1, r}+\ldots+j_{p, r}
$$

where $r \in\{0,1, \ldots, p\}$, the numbers of blocks labelled by $\left(r, r^{*}\right)$ or $\left((r+1)^{*}, r+1\right)$, respectively, in the union $\sigma_{0} \cup \ldots \cup \sigma_{p}$. Note that

$$
S_{r}(\pi)=\left\{\begin{array}{ll}
j_{r}(\pi)-1 & \text { if } r \geqslant 1 \\
j_{0}(\pi) & \text { if } r=0
\end{array},\right.
$$

which leads to the recurrence formula

$$
N_{0}(k, \mathbf{j})=\sum_{k_{0}+\ldots+k_{p}=k-1} \sum_{\substack{\mathbf{j}_{0}, \ldots, \mathbf{j}_{p} \\ S_{0}=j_{0}, S_{1}=j_{1}-1, \ldots, S_{p}=j_{p}-1}} \prod_{i=0}^{p} N_{i}\left(k_{i}, \mathbf{j}_{i}\right)
$$

for $k \geqslant 1$, where $\mathbf{j}_{i}=\left(j_{i, 0}, \ldots, j_{i, p}\right)$ and $S_{r}=j_{0, r}+j_{1, r}+\ldots+j_{p, r}$. Using this formula, we get

$$
\begin{aligned}
\mathcal{N}_{0}(x, \mathbf{d})-1 & =\sum_{k=0}^{\infty} \sum_{\mathbf{j}} N_{0}(k+1, \mathbf{j}) \mathbf{d}^{\mathbf{j}} x^{k+1} \\
& =\sum_{k=0}^{\infty} \sum_{k_{0}+\ldots+k_{p}=k} \sum_{\substack{S_{0}=j_{0}, S_{1}, j_{1}, \ldots, \mathbf{j}_{p}, \ldots, S_{p}=j_{p}-1 \\
i=0}} \prod_{i=0}^{p} N_{i}\left(k_{i}, \mathbf{j}_{i}\right) \mathbf{d}^{\mathbf{j}} x^{k+1} \\
& =x d_{1} d_{2} \ldots d_{p} \sum_{k=0}^{\infty} \sum_{\substack{\mathbf{j}, \mathbf{j}_{0}, \ldots, \mathbf{j}_{p} \\
k_{0}+\ldots+k_{p}=k}} \prod_{i=0}^{p} N_{i}\left(k_{i}, \mathbf{j}_{i}\right) \mathbf{d}^{\mathbf{j}} x^{k} \\
& =x d_{1} d_{2} \ldots d_{p} \prod_{i=0}^{p} \mathcal{N}_{i}(x, \mathbf{d})
\end{aligned}
$$

which proves our assertion. 


\section{Main results}

The main result of this paper consists in demonstrating that the multivariate FussNarayana polynomials $F_{k}$ defined in Section 2 are special cases of the polynomials $P_{k}$ obtained in [8] as limit moments of products of independent random matrices. This fact is proved in the theorem given below. We also show that the polynomials $F_{k}$ are moments of free multiplicative convolutions of Marchenko-Pastur distributions with arbitrary shape parameters.

Theorem 11. The coefficients of the polynomial $P_{k}$ are generalized Fuss-Narayana numbers, namely

$$
N_{0}(k, \mathbf{j})=\frac{1}{k}\left(\begin{array}{c}
k \\
j_{0}+1
\end{array}\right)\left(\begin{array}{l}
k \\
j_{1}
\end{array}\right) \ldots\left(\begin{array}{l}
k \\
j_{p}
\end{array}\right)
$$

where $\mathbf{j}=\left(j_{0}, j_{1}, \ldots, j_{p}\right)$, whenever $k \geqslant 1$ and $j_{0}+\ldots+j_{p}=p k$, with $N_{0}(0,0, \ldots, 0)=1$. In other cases, $N_{0}(k, \mathbf{j})=0$.

Proof. By Lemmas 9 and 10, we have

$$
d_{0}\left(\mathcal{N}_{0}(x, \mathbf{d})-1\right)=x \prod_{i=0}^{p}\left(d_{i}\left(\mathcal{N}_{0}(x, \mathbf{d})-1\right)+d_{i}\right),
$$

which means that the function of the form

$$
g(x)=d_{0}\left(\mathcal{N}_{0}(x, \mathbf{d})-1\right)
$$

is a solution of the equation (2). Hence, by Proposition 5

$$
d_{0}\left(\mathcal{N}_{0}(x, \mathbf{d})-1\right)=\mathcal{N}(x, \mathbf{d}) .
$$

Thus,

$$
\begin{aligned}
\sum_{k=1}^{\infty} \sum_{j_{0}, \ldots, j_{p}=0}^{\infty} N_{0}(k, \mathbf{j}) \mathbf{d}^{\mathbf{j}} x^{k} & =\sum_{k=1}^{\infty} \sum_{j_{0}+\ldots+j_{p}=p k+1} N\left(k, j_{0}, \ldots, j_{p}\right) d_{0}^{j_{0}-1} d_{1}^{j_{1}} \ldots d_{p}^{j_{p}} x^{k} \\
& =\sum_{k=1}^{\infty} \sum_{j_{0}+\ldots+j_{p}=p k} N\left(k, j_{0}+1, \ldots, j_{p}\right) \mathbf{d}^{\mathbf{j}} x^{k}
\end{aligned}
$$

since the generalized Fuss-Narayana numbers are defined for $j_{0}, \ldots, j_{p} \in \mathbb{N}$ and thus one can divide $\mathcal{N}(x, \mathbf{y})$ by $y_{0}$ and then replace the summation index $j_{0}$ by $j_{0}+1$. Therefore, in the last summation, $j_{0} \in \mathbb{N} \cup\{0\}$. Of course, also $j_{i} \in \mathbb{N} \cup\{0\}$ for $i>0$, with the restriction that $j_{0}+\ldots+j_{p}=k p$ and $j_{0}, \ldots, j_{p} \leqslant k$, which means that only one index among these may be equal to zero. Comparing the coefficients, we obtain our assertion for $k \geqslant 1$. The case $k=0$ is obvious.

Corollary 12. For any $k, p \in \mathbb{N}$, it holds that

$$
F_{k}\left(t_{1}, t_{2}, \ldots, t_{p}\right)=P_{k}\left(1, t_{1}, \ldots, t_{p}\right) .
$$


Proof. By Theorem 11, $d_{0} P_{k}\left(d_{0}, d_{1}, \ldots, d_{p}\right)=R_{k}\left(d_{0}, d_{1}, \ldots, d_{p}\right)$ and thus, using Remark 3 , we get the desired formula.

Example 13. In Example 8, the polynomials $P_{2}$ and $P_{3}$ were calculated for $p=2$ by means of Definition 7. Now, we can calculate them using Theorem 11. We have

$$
P_{k}\left(d_{0}, d_{1}, d_{2}\right)=\sum_{j_{0}+j_{1}+j_{2}=p k} N_{0}\left(k, j_{0}, j_{1}, j_{2}\right) d_{0}^{j_{0}} d_{1}^{j_{2}} d_{2}^{j_{3}} .
$$

For $k=2$, we obtain $N_{0}(2,0,2,2)=N_{0}(2,1,1,2)=N_{0}(2,1,2,1)=1$ and thus

$$
F_{2}\left(t_{1}, t_{2}\right)=t_{1}^{2} t_{2}^{2}+t_{1} t_{2}^{2}+t_{1}^{2} t_{2}
$$

is the corresponding multivariate Fuss-Narayana polynomial. In turn, for $k=3$, we obtain $N_{0}(3,0,3,3)=N_{0}(3,2,1,3)=N_{0}(3,2,3,1)=1$ and $N_{0}(3,2,2,2)=N_{0}(3,1,2,3)=$ $N_{0}(3,1,3,2)=3$ and thus

$$
F_{3}\left(t_{1}, t_{2}\right)=t_{1}^{3} t_{2}^{3}+t_{1} t_{2}^{3}+t_{1}^{3} t_{2}+3 t_{1}^{2} t_{2}^{2}+3 t_{1}^{2} t_{2}^{3}+3 t_{1}^{3} t_{2}^{2}
$$

is the corresponding mutlivariate Fuss-Narayana polynomial.

The next corollary is an easy application of the above theorem and of the Vandermonde's identity. The original proof of this fact is due to Kemp and Speicher [6].

Corollary 14. The cardinality of the set $\mathcal{N C}_{2 p k}^{2}\left(W_{0}^{k}\right)$ is the Fuss-Catalan number, i.e.

$$
\left|\mathcal{N C}_{2 p k}^{2}\left(W_{0}^{k}\right)\right|=\frac{1}{p k+1}\left(\begin{array}{c}
(p+1) k \\
k
\end{array}\right) .
$$

Proof. It is enough to put $d_{0}=d_{1}=\ldots=d_{p}=1$ in (3).

Finally, let us show a direct application of multivariate Fuss-Catalan polynomials to free probability. Denote by

$$
\varrho_{t}=\max \{1-t, 0\} \delta_{0}+\frac{\sqrt{(x-a)(b-x)}}{2 \pi x} \mathbb{1}_{[a, b]}(x) d x
$$

where $a=(1-\sqrt{t})^{2}$ and $b=(1+\sqrt{t})^{2}$, the Marchenko-Pastur law with the shape parameter equal to $t>0$. This distribution plays the role of the free analog of the Poisson law and is often called the free Poisson law [11].

We will prove that the moments of free multiplicative convolutions

$$
\varrho_{t_{1}} \otimes \varrho_{t_{2}} \otimes \ldots \otimes \varrho_{t_{p}}
$$

of Marchenko-Pastur laws with different shape parameters $t_{1}, t_{2}, \ldots, t_{p}$ are multivariate Fuss-Narayana polynomials. An explicit formula for the much simpler case when all shape parameters are equal (and thus we deal with convolution powers of $\varrho_{t}$ ) has recently been found by Hinz and Młotkowski [5]. Another special case of our formula is that of FussNarayana polynomials, obtained for $t_{1}=\ldots=t_{p-1}=1$ and $t_{p}=t$, which gives the moments of free Bessels laws $\pi_{p, t}$ of Banica et al [3, Theorem 5.2]. 
Proposition 15. For any positive $t_{1}, t_{2}, \ldots, t_{p}$ and $k \in \mathbb{N}$, it holds that

$$
m_{k}\left(\varrho_{t_{1}} \otimes \varrho_{t_{2}} \otimes \ldots \otimes \varrho_{t_{p}}\right)=F_{k}\left(t_{1}, t_{2}, \ldots, t_{p}\right) .
$$

Proof. Let $S_{\mu}$ and $\psi_{\mu}$ denote the S-transform and the moment generating function (without the constant term) of a probability measure on the real line $\mu$, respectively. It is well-known that

$$
S_{\mu}(z)=\frac{1+z}{z} \psi_{\mu}^{-1}(z)
$$

where $\psi_{\mu}^{-1}$ is the composition inverse of $\psi_{\mu}$. Moreover, the S-transform of the MarchenkoPastur law is

$$
S_{\varrho_{t}}(z)=\frac{1}{z+t}
$$

for any $t>0$. Using the mutliplicativity of the S-transform with respect to the free multiplicative convolution, we obtain

$$
\psi_{\varrho}^{-1}(z)=\frac{1}{(z+1)\left(z+t_{1}\right) \ldots\left(z+t_{p}\right)}
$$

where $\varrho=\varrho_{t_{1}} \otimes \varrho_{t_{2}} \otimes \ldots \otimes \varrho_{t_{p}}$. Obviously, the above formula is equivalent to

$$
\psi_{\varrho}(z)=z\left(\psi_{\varrho}(z)+1\right)\left(\psi_{\varrho}(z)+t_{1}\right) \ldots\left(\psi_{\varrho}(z)+t_{p}\right) .
$$

Now, using Proposition 5 with $d_{0}=1, d_{1}=t_{1}, \ldots, d_{p}=t_{p}$, we obtain the desired formula.

Example 16. $m_{2}\left(\varrho_{t_{1}} \otimes \varrho_{t_{2}} \otimes \varrho_{t_{3}}\right)=t_{1}^{2} t_{2}^{2} t_{3}^{2}+t_{1} t_{2}^{2} t_{3}^{2}+t_{1}^{2} t_{2} t_{3}^{2}+t_{1}^{2} t_{2}^{2} t_{3}$.

\section{References}

[1] N. Alexeev, F. Götze, and A. Tikhomirov. Asymptotic distribution of singular values of powers of random matrices. Lithuanian Math. J., 50:121-132, 2010.

[2] N. Alexeev, F. Götze, and A. Tikhomirov. On the asymptotic distributions of singular values of products of large rectangular random matrices. arXiv:1012.2586 [math. PR], 2011.

[3] T. Banica, S. T. Belinschi, M. Capitaine, and B. Collins. Free Bessel laws. Canad. J. Math., 63:3-37, 2011.

[4] E. Deutsch. Dyck path enumeration. Discrete Math. 204:167-202, 1999.

[5] M. Hinz and W. Młotkowski. Free powers of the free Poisson measure. Colloq. Math., 123:285-290, 2011.

[6] T. Kemp and R. Speicher. Strong Haagerup inequalities for free R-diagonal elements. J. Funct. Anal., 251:141-173, 2007.

[7] R. Lenczewski. Asymptotic properties of random matrices and pseudomatrices. Adv. Math., 228:2403-2440, 2011. 
[8] R. Lenczewski. Limit distributions of random matrices. arXiv:1208.3586 [math. OA], 2012.

[9] S. Loktev. Weight multiplicity polynomials of multi-variable Weyl modules, 2nd ed. Mosc. Math. J., 10(1):215-229, 2010.

[10] V. Marchenko and L. Pastur. The distribution of eigenvalues in certain sets of random matrices. Math. Sb. 72:507-536, 1967.

[11] D. Voiculescu, K. Dykema, and A. Nica. Free random variables. CRM Monograph Series, no. 1. A.M.S., Providence, 1992.

[12] H. S. Wilf, Generatingfunctionology, 2nd ed.. Academic Press, New York, 1994. 